VOL. 45 (1992) [277-283]

\title{
THE MOMENT SPACES OF NORMED LINEAR SPACES
}

\section{Fowzi Ahmad Sejeeni and Matoog Ahmad Badri}

For a linearly independent sequence in a normed linear space the moment space is defined. Basic properties of moment spaces are discussed as well as a necessary and sufficient condition for the moment space to be a closed subspace of $\ell^{\infty}$.

\section{INTRODUCTION}

There is a whole variety of classical problems of moments, variously known as the Hausdorff moment problem (the little moment problem) and the Hamburger moment problem (see $[1$, p.349] and $[3$, p.197]). The last one can be stated as follows. Let $\mu$ be a positive Borel measure on $\mathbb{R}$ such that $\int|t|^{n} d \mu(t)=a_{n}<\infty$ for $n \geqslant 1$. The sequence $\left\{a_{n}\right\}$ is called the moment sequence of $\mu$. The moment problem here is to characterise those sequences of numbers that are moment sequences.

In this paper, we define moment spaces in the more general setting of normed linear spaces and study their basic properties.

First, we establish our notations and definitions.

For a normed linear space $E$ we shall denote by $E^{*}$ its dual, the space of all bounded linear functionals on $E$. For a sequence $\left\{\chi_{n}\right\}$ in $E$ we shall denote by $\left[\chi_{n}\right]$ the closed linear span of $\left\{\chi_{n}\right\}$; that is, $\left[\chi_{n}\right]=\overline{\operatorname{span}\left\{\chi_{n}\right\}}$. The sequence $\left\{\chi_{n}\right\}$ is called fundamental if $\left[\chi_{n}\right]=E$. A sequence $\left\{\chi_{n}\right\}$ in a Banach space $E$ is called a (Schauder) basis if, for any $\chi \in E$, there exists a unique sequence $\left\{a_{n}\right\}$ of scalars such that $\chi=\sum_{n=1}^{\infty} a_{n} \chi_{n}$. The sequence $\left\{\chi_{n}\right\}$ is a basic sequence in $E$ if $\left\{\chi_{n}\right\}$ is a basis for $\left[\chi_{n}\right]$. Let $\left\{\chi_{n}\right\}$ be a basis for a Banach space $E$ and let $f_{n}: E \rightarrow \mathbb{C}$ be defined by $f_{n}\left(\sum_{k=1}^{\infty} a_{k} \chi_{k}\right)=a_{n}$ for $n=1,2,3, \ldots$ Then each $f_{n} \in E^{*}$ and $\left\{f_{n}\right\}_{n=1}^{\infty}$ is called the sequence of coefficient functionals associated with the basis $\left\{\chi_{n}\right\}$ (see [2], p.32). For normed linear spaces $E$ and $Y, f: E \rightarrow Y$ is a linear isomorphism if $f$ is a vector-space isomorphism and a homeomorphism onto $Y$. As usual $\ell^{\infty}$ is the Banach space of all bounded complex sequences $a=\left\{a_{n}\right\}$ with $\|a\|_{\infty}=\sup _{n}\left|a_{n}\right|, c$ denotes the space of all convergent sequences with $\|\cdot\|_{\infty}$, and $c_{0}=\left\{\left\{a_{n}\right\}: \lim _{n} a_{n}=0\right\}$. Note that $c$ and

\section{Received 9 April 1991}

Copyright Clearance Centre, Inc. Serial-fee code: 0004-9729/92 \$A2.00+0.00. 
$c_{0}$ are closed subspaces of $\ell^{\infty}$. For $1 \leqslant p<\infty, \ell_{p}^{\infty}$ denotes the space of all sequences $\left\{a_{n}\right\}$ such that $\sum_{n=1}^{\infty}\left|a_{n}\right|^{p}<\infty$ with the norm $\|\cdot\|_{\infty}$, and $\ell_{p}$ denotes the same space with the usual norm $\|a\|_{p}=\left(\sum_{n=1}^{\infty}\left|a_{n}\right|^{p}\right)^{1 / p}$. We say that the sequences $\left\{\chi_{n}\right\}$ in $E$ and $\left\{y_{n}\right\}$ in $Y$ are equivalent if there exists a linear isomorphism $T$ of $E$ onto $Y$ such that $T \chi_{n}=y_{n}$ for all $n$.

Now, we are ready to define moment spaces.

Definition 1.1: Let $E$ be a normed linear space and $\left\{\chi_{n}\right\}$ a linearly independent sequence in $E$. We define $M\left(E,\left\{\chi_{n}\right\}\right)$ (the moment space of $E$ with respect to $\left\{\chi_{n}\right\}$ ) by $M\left(E,\left\{\chi_{n}\right\}\right)=\left\{\left\{f\left(\chi_{n}\right)\right\}_{n=1}^{\infty}: f \in E^{*}\right\}$. Each element of $M\left(E,\left\{\chi_{n}\right\}\right)$ is called a moment sequence.

Clearly, $M\left(E,\left\{\chi_{n}\right\}\right)$ is a non-trivial vector space. Suppose $\left\{\chi_{n}\right\}_{n=1}^{\infty}$ is bounded; that is, $\sup _{n}\left\|\chi_{n}\right\|<\infty$. Let $a=\left\{a_{n}\right\} \in M\left(E,\left\{\chi_{n}\right\}\right)$ and $f \in E^{*}$ be such that $f\left(\chi_{n}\right)=a_{n}$. Then $\sup _{n}\left|a_{n}\right|=\sup _{n}\left|f\left(\chi_{n}\right)\right| \leqslant\left(\sup _{n}\left\|\chi_{n}\right\|\right)\|f\|<\infty$. Thus $a \in \ell^{\infty}$ and hence if $\left\{\chi_{n}\right\}$ is bounded, we will regard $M\left(E,\left\{\chi_{n}\right\}\right)$ as a subspace of $\ell^{\infty}$.

In the next section, we start by showing that $M\left(E,\left\{\chi_{n}\right\}\right)$ is a continuous linear image of $E^{*}$ (Theorem 2.1). In Theorem 2.3, we prove a simple but useful fact that $M\left(E,\left\{\chi_{n}\right\}\right)=M\left(\left[\chi_{n}\right],\left\{\chi_{n}\right\}\right)$. We then compute some moment spaces. In Theorem 2.6, we study the relation between equivalent sequences of Banach spaces and their moment spaces. In Theorem 2.8, we prove the following: Let $E$ be a Banach space with a bounded basis $\left\{\chi_{n}\right\}$ and let $\left\{f_{n}\right\}$ be the associated sequence of coefficient functionals, then $M\left(E,\left\{\chi_{n}\right\}\right)$ is closed in $\ell^{\infty}$ if and only if $\sum_{n=1}^{\infty}\left|f_{n}(\chi)\right|<\infty$ for each $\chi \in E$. The last two theorems present different sets of conditions that ensure that the moment spaces are $c_{0}, \ell_{1}$, or $\ell^{\infty}$.

\section{Results}

TheOREM 2.1. Let $E$ be a normed linear space and $\left\{\chi_{n}\right\}$ a bounded linearly independent sequence in $E$. Define $\Gamma: E^{*} \rightarrow \ell^{\infty}$ by $\Gamma(f)(n)=f\left(\chi_{n}\right)$ for all $n$; then $\Gamma$ is a continuous linear map onto $M\left(E,\left\{\chi_{n}\right\}\right)$.

Proof: Let $f, g \in E^{*}$ and $\alpha \in \mathbb{C}$. Then $\Gamma(\alpha f+g)(n)=(\alpha f+g)\left(\chi_{n}\right)=$ $\alpha f\left(\chi_{n}\right)+g\left(\chi_{n}\right)=\alpha \Gamma(f)(n)+\Gamma(g)(n)$. Also for $f \in E^{*}$ we have $\|\Gamma(f)\|=\sup _{n}\left|f\left(\chi_{n}\right)\right| \leqslant$ $\left(\sup _{n}\left\|\chi_{n}\right\|\right)\|f\|$. Hence $\Gamma$ is a continuous linear map onto $M\left(E,\left\{\chi_{n}\right\}\right)$.

Corollary 2.2. Assume the hypothesis of Theorem 2.1. If $\left\{\chi_{n}\right\}$ is fundamental, then $\Gamma$ is an injection. 
Proof: Let $f \in \operatorname{Ker} \Gamma$, then $\Gamma(f)(n)=f\left(\chi_{n}\right)=0$ for all $n \in \mathbb{N}$. Hence, we have $f(\chi)=0$ for each $\chi \in E$; that is $f \equiv 0$.

Before proceeding further, we prove a simple but useful result.

THEOREM 2.3. Let $E$ be a normed linear space and $\left\{\chi_{n}\right\}$ a linearly independent sequence in $E$. Then $M\left(E,\left\{\chi_{n}\right\}\right)=M\left(\left[\chi_{n}\right],\left\{\chi_{n}\right\}\right)$.

Proof: Let $a \in M\left(E,\left\{\chi_{n}\right\}\right)$ and $f \in E^{*}$ be such that $f\left(\chi_{n}\right)=a_{n}$. But then $\left.f\right|_{\left[\chi_{n}\right]} \in\left[\chi_{n}\right]^{*}$, implies that $a \in M\left(\left[\chi_{n}\right],\left\{\chi_{n}\right\}\right)$. The converse follows by the HahnBanach extension theorem.

REMARK. It follows from the last theorem that, in order to study $M\left(E,\left\{\chi_{n}\right\}\right)$, we may assume that $\left\{\chi_{n}\right\}$ is fundamental.

Definition 2.4: A squence $\left\{\chi_{n}\right\}$ in normed linear space is called regular if it is bounded, linearly independent and fundamental. Note that a bounded basis is always regular.

Let $e_{n}=\left\{\delta_{n k}\right\}_{k=1}^{\infty}$ for $n=1,2,3, \ldots$ It is known that $\left\{e_{n}\right\}$ is a regular basis for $c_{0}$ and for $\ell_{p}(1 \leqslant p<\infty)$.

Proposition 2.5. We have

(a) $M\left(c_{0},\left\{e_{n}\right\}\right)=\ell_{1}^{\infty} ;$

(b) $M\left(\ell_{1},\left\{e_{n}\right\}\right)=\ell^{\infty}$;

(c) $M\left(\ell^{\infty},\left\{e_{n}\right\}\right)=M\left(c,\left\{e_{n}\right\}\right)=\ell_{1}^{\infty}$;

(d) $M\left(\ell_{p},\left\{e_{n}\right\}\right)=\ell_{q}^{\infty}$,

(where $1<p<\infty$ and $q$ its conjugate exponent).

Proof: (a) From Riesz representation theorem we have $c_{0}^{*}=\ell_{1}$. Now apply Corollary 2.2 to get $M\left(c_{0},\left\{e_{n}\right\}\right)=\ell_{1}^{\infty}$ (since $\left\{e_{n}\right\}$ is regular).

(b) Similar.

(c) Since $c_{0}$ is a closed subspace of $\ell^{\infty}$ and of $c$ and $\left[e_{n}\right]=c_{0}$, we have $M\left(\ell^{\infty},\left\{e_{n}\right\}\right)=M\left(c,\left\{e_{n}\right\}\right)=M\left(c_{0},\left\{e_{n}\right\}\right)=\ell_{1}^{\infty}$, by Theorem 2.3.

(d) Similar to part (a).

The following theorem links up the moment spaces with the equivalence of sequences in Banach spaces.

Theorem 2.6. Let $E$ and $Y$ be Banach spaces and $\left\{\chi_{n}\right\},\left\{y_{n}\right\}$ regular sequences in $E, Y$ respectively. If the two sequences are equivalent, then $M\left(E,\left\{\chi_{n}\right\}\right)=$ $M\left(Y,\left\{y_{n}\right\}\right)$. Conversely, if $M$ is a closed subspace of $\ell^{\infty}$ and $M\left(E,\left\{\chi_{n}\right\}\right)=$ $M\left(Y,\left\{y_{n}\right\}\right)=M$ then $\left\{\chi_{n}\right\},\left\{y_{n}\right\}$ are equivalent.

Proof: Suppose $T: E \rightarrow Y$ is an isomorphism such that $T\left(\chi_{n}\right)=y_{n}$ for all $n$. Let $a=\left\{a_{n}\right\} \in M\left(Y,\left\{y_{n}\right\}\right)$ and $g \in Y^{*}$ such that $g\left(y_{n}\right)=a_{n}$. Then $g \circ T \in E^{*}$ 
and $(g \circ T)\left(\chi_{n}\right)=a_{n}$, for all $n$. Hence, $a \in M\left(E,\left\{\chi_{n}\right\}\right)$ and so $M\left(Y,\left\{y_{n}\right\}\right) \subseteq$ $M\left(E,\left\{\chi_{n}\right\}\right)$. The reverse inclusion follows similarly.

Conversely, let $\Gamma_{1}: E^{*} \rightarrow M$, and $\Gamma_{2}: Y^{*} \rightarrow M$ be the linear isomorphisms that follow from Corollary 2.2 and the fact that $M$ is closed. Let $T=\left(\Gamma_{1}^{-1} \circ \Gamma_{2}\right)^{*}$ (where * denotes the Banach space adjoint). Then $T$ is a linear isomorphism of $E^{* *}$ onto $Y^{* *}$. We will show that $T$ restricted to $E$ is a linear isomorphism of $E$ onto $Y$ with $T\left(\chi_{n}\right)=y_{n}, n \in \mathbb{N}$ (where $E, Y$ are regarded as closed subspaces of their second duals via the canonical embeddings). Now, $T\left(\chi_{n}\right)=\left(\Gamma_{2}^{*} \circ\left(\Gamma_{1}^{-1}\right)^{*}\right) \chi_{n}=\Gamma_{2}^{*}\left(\chi_{n} \circ \Gamma_{1}^{-1}\right)=$ $\left(\chi_{n} \circ \Gamma_{1}^{-1} \circ \Gamma_{2}\right)$. Hence, for all $g \in Y^{*}$,

$$
\begin{aligned}
T\left(\chi_{n}\right)(g) & =\left(\chi_{n} \circ \Gamma_{1}^{-1} \circ \Gamma_{2}\right)(g)=\left(\chi_{n} \circ \Gamma_{1}^{-1}\right)\left\{g\left(y_{k}\right)\right\}_{k=1}^{\infty} \\
& =\left(\chi_{n} \circ \Gamma_{1}^{-1}\right)\left\{f\left(\chi_{k}\right)\right\}_{k=1}^{\infty}=\chi_{n}(f)=f\left(\chi_{n}\right)=g\left(y_{n}\right)
\end{aligned}
$$

for some $f \in E^{*}$ (since $\left.M\left(E,\left\{\chi_{n}\right\}\right)=M\left(Y,\left\{y_{n}\right\}\right)\right)$. Thus $T\left(\chi_{n}\right)(g)=y_{n}(g)$ for all $g \in Y^{*}$; that is, $T\left(\chi_{n}\right)=y_{n}$. Next, $T$ is linear, so $T\left(\operatorname{span}\left\{\chi_{n}\right\}\right)=\operatorname{span}\left\{y_{n}\right\}$ and $T\left(\left[\chi_{n}\right]\right)=\overline{T\left(\operatorname{span}\left\{\chi_{n}\right\}\right)}$ (since $T$ is a homeomorphism). Hence, $T(E)=T\left(\left[\chi_{n}\right]\right)=$ $\overline{T\left(\operatorname{span}\left\{\chi_{n}\right\}\right)}=\overline{\operatorname{span}\left\{y_{n}\right\}}=Y$.

The closeness assumption in Theorem 2.6 is essential as we can see in the following: Let $E=\ell^{\infty}, Y=c$; then $M\left(\ell^{\infty},\left\{e_{n}\right\}\right)=M\left(c,\left\{e_{n}\right\}\right)=\ell_{1}^{\infty}$ while $\left\{e_{n}\right\}$ in $E$ is not equivalent to $\left\{e_{n}\right\}$ in $Y$.

The next important and known result will be used in the sequel.

THEOREM 2.7. (See $[2$, p.44]). The following statements (regarding a formal series $\sum_{k=1}^{\infty} z_{k}$ in a Banach space $E$ ) are equivalent:

(i) $\sum_{k=1}^{\infty}\left|f\left(z_{k}\right)\right|<\infty$ for all $f \in E^{*}$;

(ii) there is a constant $K>0$ such that for each $a \in \ell^{\infty}$,

$$
\sup _{n}\left\|\sum_{k=1}^{n} a_{k} z_{k}\right\| \leqslant K\|a\|_{\infty} ;
$$

(iii) for any $c \in c_{0}, \sum_{k=1}^{\infty} c_{k} z_{k}$ converges.

THEOREM 2.8. Let $E$ be a Banach space, $\left\{\chi_{n}\right\}$ a bounded basis for $E$ and $\left\{f_{n}\right\}$ the associated sequence of coefficient functionals. Then the following are equivalent:

(i) $\sum_{n=1}^{\infty}\left|f_{n}(\chi)\right|<\infty$ for each $\chi \in E$;

(ii) $M\left(E,\left\{\chi_{n}\right\}\right)$ is a closed subspace of $\ell^{\infty}$ (in the $\|\cdot\|_{\infty}$ norm). 
Proof: (i) implies (ii):

Let $\left\{a^{m}\right\}_{m=1}^{\infty}$ be a sequence in $M\left(E,\left\{\chi_{n}\right\}\right)$ converging to $a$ in $\ell^{\infty}$ (where $a^{m}=$ $\left\{a_{n}^{m}\right\}_{n=1}^{\infty}$ and $\left.a=\left\{a_{n}\right\}_{n=1}^{\infty}\right)$ and $\left\{g_{m}\right\}$ the corresponding element of $E^{*}$ such that $g_{m}\left(\chi_{n}\right)=a_{n}^{m}$.

For $\chi=\sum_{n=1}^{\infty} f_{n}(\chi) \chi_{n}$ define $f(\chi)=\sum_{n=1}^{\infty} a_{n} f_{n}(\chi)$. The series $\sum_{n=1}^{\infty} a_{n} f_{n}(\chi)$ converges absolutely (since $\sum_{n=1}^{\infty} a_{n}\left|f_{n}(\chi)\right| \leqslant\|a\|_{\infty} \sum_{n=1}^{\infty}\left|f_{n}(\chi)\right|<\infty$ ). Hence $f$ is a linear functional on $E$ and $f\left(\chi_{n}\right)=a_{n}$ for all $n$. Also for all $\chi \in E$, we have

$$
\begin{aligned}
\left|g_{m}(\chi)-f(\chi)\right| & =\left|\sum_{n=1}^{\infty}\left(a_{n}^{m}-a_{n}\right) f_{n}(\chi)\right| \\
& \leqslant\left(\left\|a_{n}^{m}-a_{n}\right\|_{\infty} \sum_{n=1}^{\infty}\left|f_{n}\left(\chi_{m}\right)\right|\right) \rightarrow 0 .
\end{aligned}
$$

Thus by Banach-Steinhaus theorem, $f \in E^{*}$, and then $a \in M\left(E,\left\{\chi_{n}\right\}\right)$.

(ii) implies (i): The operator $\Gamma: E^{*} \rightarrow M\left(E,\left\{\chi_{n}\right\}\right)$, defined in Theorem 2.1 is a linear isomorphism, so by the inverse mapping theorem there exists a constant $K>0$ such that $\|f\| \leqslant K\|\Gamma(f)\|_{\infty}$. For $a=\left\{a_{n}\right\} \in \ell^{\infty}$, we have $\left\|\sum_{k=1}^{n} a_{k} f_{k}\right\| \leqslant K\left\|\sum_{k=1}^{n} \Gamma\left(f_{k}\right)\right\|_{\infty}=$ $K\left\|\left(a_{1}, a_{2}, \ldots, a_{n}, 0, \ldots\right)\right\|_{\infty} \leqslant K\|a\|_{\infty}$. Hence, $\sup _{n}\left\|\sum_{k=1}^{n} a_{k} f_{k}\right\| \leqslant K\|a\|_{\infty}$, and the conclusion follows by Theorem 2.7.

Corollary 2.9. Let $E$ and $Y$ be Banach spaces. If $\left\{\chi_{n}\right\}$ and $\left\{y_{n}\right\}$ are equivalent basic sequences in $E$ and $Y$ respectively and $\left\{f_{n}\right\},\left\{g_{n}\right\}$ their respective coefficient functionals, then $\sum_{k=1}^{\infty}\left|f_{k}(\chi)\right|<\infty$ for all $\chi \in E$ if and only if $\sum_{k=1}^{\infty}\left|g_{k}(y)\right|<\infty$ for all $y \in Y$.

Proof: Combine Theorem 2.6 with Theorem 2.8.

Proposition 2.10. Let $E$ denote any of the following Banach spaces $c_{0}, c$, or $\ell_{p}(1 \leqslant p \leqslant \infty)$ and let $\left\{e_{n}\right\}$ be the standard unit vector basis. Then $M\left(E,\left\{e_{n}\right\}\right)$ is closed in $\ell^{\infty}$ if and only if $E=\ell_{1}$.

Proof: First, if $E$ denotes $c_{0}, c$ or $\ell_{\infty}$, then $M\left(E,\left\{e_{n}\right\}\right)=\ell_{1}^{\infty}$, which is not closed in $\ell^{\infty}$. Next, if $E$ denotes $\ell_{p}(1 \leqslant p \leqslant \infty)$, then $\sum_{k=1}^{\infty}\left|f_{k}(\chi)\right|<\infty$ for each $\chi=\sum_{k=1}^{\infty} f_{k}(\chi) e_{k} \in E$ (where $\left\{f_{k}\right\}$ is the associated sequence of coefficient functionals) if and only if $E=\ell_{1}$. Hence, $M\left(E,\left\{e_{m}\right\}\right)$ is closed in $\ell^{\infty}$ if and only if $E=\ell_{1}$ (Theorem 2.8). 
TheOREM 2.11. Let $E$ be a Banach space, $\left\{\chi_{n}\right\}$ a bounded basis for $E$ and $\left\{f_{n}\right\}$ the associated sequence of coefficient functionals. If $\left\{f_{n}\right\}$ is a basis for $E^{*}$ and $\sum_{n=1}^{\infty}\left|\phi\left(f_{n}\right)\right|<\infty$ for all $\phi \in E^{* *}$, then

(i) $M\left(E,\left\{\chi_{n}\right\}\right)=c_{0}$;

(ii) $M\left(E^{*},\left\{f_{n}\right\}\right)=\ell_{1}^{\infty}$.

Proof: (i) Let $a \in M\left(E,\left\{\chi_{n}\right\}\right)$ and $f \in E^{*}$ be such that $f\left(\chi_{n}\right)=a_{n}$ for all $n$. Write $f=\sum_{n=1}^{\infty} d_{n} f_{n}$ (since $\left\{f_{n}\right\}$ is a basis for $E^{*}$ ). Then $f\left(\chi_{k}\right)=$ $\sum_{n=1}^{\infty} d_{n} f_{n}\left(\chi_{n}\right)=d_{k}$ for all $k$. Now by Theorem 2.7 we have $\left|a_{n}\right|\left\|f_{n}\right\|=\left\|f\left(\chi_{n}\right) f_{n}\right\|=$ $\left\|\sum_{k=1}^{n} f\left(\chi_{k}\right) f_{k}-\sum_{k=1}^{n-1} f\left(\chi_{k}\right) f_{k}\right\| \vec{n} 0$. Also, $\inf _{n}\left\|f_{n}\right\|>0$ (since $1=\left|f_{n}\left(\chi_{n}\right)\right| \leqslant$ $\left.\left\|f_{n}\right\|\left\|\chi_{n}\right\|\right)$, so $a_{n} \rightarrow 0$; that is, $a \in c_{0}$.

Conversely, let $c \in c_{0}$; the series $\sum_{n=1}^{\infty} c_{n} f_{n}$ converges in $E^{*}$ by Theorem 2.7 (since $\sum_{n=1}^{\infty}\left|\phi\left(f_{n}\right)\right|<\infty$ for all $\left.\phi \in E^{* *}\right)$. Write $f=\sum_{n=1}^{\infty} c_{n} f_{n} ;$ then $f\left(\chi_{k}\right)=\sum_{k=1}^{\infty} c_{n} f_{n}\left(\chi_{k}\right)=$ $c_{k}$. Thus $c \in M\left(E,\left\{\chi_{n}\right\}\right)$.

(ii) First, observe that the hypothesis above implies that $\left\{f_{n}\right\}$ is bounded. Now, let $a \in M\left(E^{*},\left\{f_{n}\right\}\right)$ and $\phi \in E^{* *}$ be such that $\phi\left(f_{n}\right)=a_{n}$. To show $a \in \ell_{1}^{\infty}$, it suffices to show $\sum_{k=1}^{\infty} b_{k} a_{k}$ converges for all $b \in c_{0}$. For, let $b \in c_{0}$; then by Theorem 2.7, $\sum_{k=1}^{\infty} b_{k} f_{k}$ converges to an element of $E^{*}$. Now $\phi\left(\sum_{k=1}^{\infty} b_{k} f_{k}\right)=\sum_{k=1}^{\infty} b_{k} \phi\left(f_{k}\right)=\sum_{k=1}^{\infty} b_{k} a_{k}$, so that $\sum_{k=1}^{\infty} b_{k} a_{k}$ converges.

For the other inclusion, let $a \in \ell_{1}^{\infty}$. Define $\phi: E^{*} \rightarrow \mathbb{C}$ by $\phi(f)=\sum_{k=1}^{\infty} a_{k} f\left(\chi_{k}\right)$. Now, $|\phi(f)| \leqslant \sum_{k=1}^{\infty}\left|a_{k}\right|\left|f\left(\chi_{k}\right)\right| \leqslant\left(\sup _{n}\left\|\chi_{n}\right\|\right)\left(\sum_{k=1}^{\infty}\left|a_{k}\right|\right)\|f\|=\left(\sup _{n}\left\|\chi_{n}\right\|\right)\|a\|_{1}\|f\| ;$ hence $\phi \in E^{* *}$, and $\phi\left(f_{n}\right)=a_{n}$. Thus $a \in M\left(E^{*},\left\{f_{n}\right\}\right)$.

Theorem 2.12. Let $E$ be a Banach space, $\left\{\chi_{n}\right\}$ a bounded basis for $E$ and $\left\{f_{n}\right\}$ the associated sequence of coefficient functionals. If there exists a constant $M>0$ such that $\sum_{k=1}^{\infty}\left|f_{k}(\chi)\right| \leqslant M\|\chi\|$, for all $\chi \in E$, then $M\left(E,\left\{\chi_{n}\right\}\right)=\ell^{\infty}$.

Proof: Let $a \in \ell^{\infty}$. Define $f(\chi)=\sum_{n=1}^{\infty} a_{n} f_{n}(\chi)$; then $f \in X^{*}$. Indeed $\|f\| \leqslant$ $M\|a\|_{\infty}$. Also $f\left(\chi_{n}\right)=a_{n}$ for all $n$. Hence, $a \in M\left(E,\left\{\chi_{n}\right\}\right)$. 


\section{REFERENCES}

[1] J.B. Conway, A course in functional analysis (Springer-Verlag, Berlin, Heidelberg, New York, 1985).

[2] J. Diestel, Sequences and series in Banach spaces (Springer-Verlag, Berlin, Heidelberg, New York, 1984).

[3] L.V. Kantorovich and G.P. Akilov, Functional analysis: second edition, translated by H.L. Silcock (Pergamon Press, New York, 1982).

Department of Mathematical Sciences

Umm Al Qurs University

PO Box 3711

Makkah

Saudi Arabia 